\title{
Intestinal obstruction after trauma laparotomy: a rare case due to colon cancer
}

Received May 30, 2021

Revised August 3, 2021

Accepted August 14, 2021

Correspondence to

Pil Young Jung

Department of Surgery, Yonsei

University Wonju College of Medicine,

Wonju Severance Christian Hospital,

Trauma Center, 20 Ilsan-ro, Wonju

26426, Korea

Tel: +82-33-741-0882

Fax: +82-33-741-0574

E-mail: surgery4trauma@yonsei.ac.kr

\section{Byungjun Song ${ }^{1}$, Pil Young Jung ${ }^{2}$}

${ }^{1}$ Department of Surgery, Kangwon National University Hospital, Kangwon National University School of Medicine, Chuncheon, ${ }^{2}$ Department of Surgery, Yonsei University Wonju College of Medicine, Wonju Severance Christian Hospital, Trauma Center, Wonju, Korea

The causes of intestinal obstruction are diverse with tissue adhesion, incarcerated hernia, and large bowel neoplasm being the most common causes. Cancer is not easy to diagnose in patients with a history of intestinal obstruction after the intraperitoneal operation following blunt trauma. Herein, we report the case of a patient who was diagnosed with colon cancer after undergoing adhesiolysis due to intestinal obstruction.

Key Words: Intestinal obstruction, Tissue adhesion, Colon neoplasm

\section{Introduction}

Postoperative bowel obstruction occurs frequently and may require emergency surgery (1). About $80 \%$ of bowel obstructions are small bowel obstructions, and the main factors are adhesion, incarcerated hernia, and neoplasm $(2,3)$. The main factors of large bowel obstruction are cancer and adhesion (3-5). However, it is uncommon not uncommon for two or more factors to occur simultaneously. We report the case of a patient who was diagnosed with colon cancer after undergoing adhesiolysis for adhesive intestinal obstruction.

\section{Case presentation}

A 59-year-old man was transferred to our trauma center for postoperative intestinal obstruction that had not improved with nonsurgical treatment for more than 3 weeks.
Four years ago, the patient had undergone emergency surgery due to hemopneumoperitoneum from a pedestrian traffic accident. Ileum perforation, cecum serosa tearing, small bowel, and cecal mesentery hematoma were identified intraoperatively, but no active bleeding was found. The patient had undergone ileum primary repair, cecal serosa primary repair, and incidental appendectomy based on the surgeon's judgment. Postoperative small bowel obstruction occurred after surgery and improved after about 50 days with nonoperative management.

Since then, there had been no history of bowel obstruction. At the time of transfer, there were no laboratory findings that suggested inflammation. Abdominal computed tomography (ACT) showed no findings suggestive of ischemia or necrosis, even though the small and large intestines were dilated; however, an abrupt narrowing site was identified at the descending-sigmoid colon junction (Fig. 1). Regarding ACT, the radiologist did not mention

(c)This is an Open Access article distributed under the terms of the Creative Commons Attribution Non-Commercial License (http://creativecommons.org/licenses/by-nc/4.0) which permits unrestricted noncommercial use, distribution, and reproduction in any medium, provided the original work is properly cited.

Copyright (C) 2021 Korean Association for Research, Procedures and Education on Trauma. All rights reserved. 

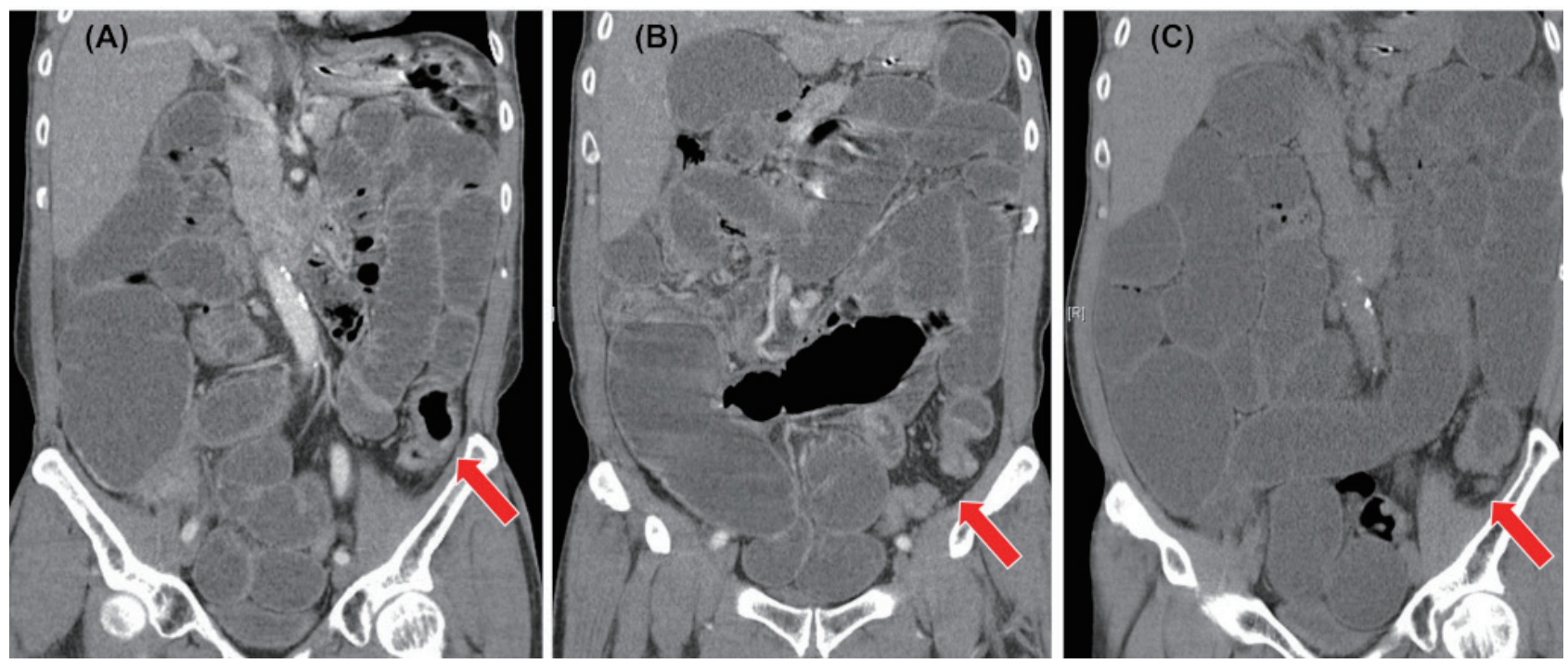

Fig. 1. Abdominal computed tomography was performed after intestinal obstruction operation. (A) admission day, (B) 10 days after, and (C) 3 weeks after. Both small and large bowels were dilated; however, the mucosal wall was enhanced well. Arrows indicate the stenotic site of the colon with mild mucosa wall thickening.

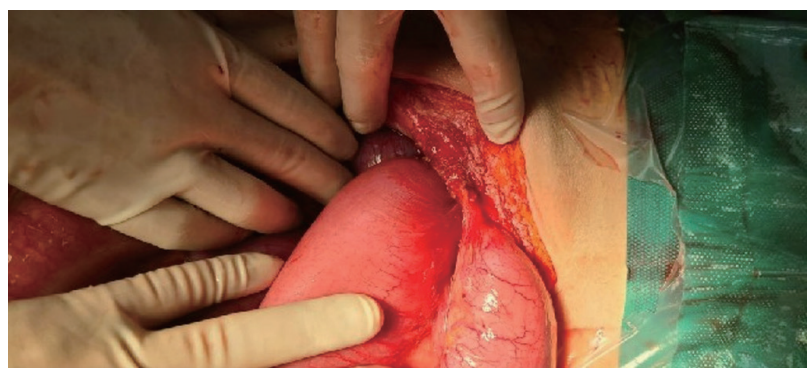

Fig. 2. A photograph was taken during the operation of adhesiolysis. The small intestine was attached to the abdominal wall in the left lower quadrant region in a V-shape. any remarkable findings other than those described above. Surgery was performed for a bowel obstruction that had not improved with a fasting period of 3 weeks or more. At the time of surgery, the small bowel that was positioned 2 $\mathrm{cm}$ below the Treitz ligament, which was considered to be the obstruction end-point, was attached to the abdominal wall in a V-shape in the left lower quadrant (Fig. 2). The overall adhesion between the small intestine and the mesentery was checked, and the evaluation of the descending colon was limited to the adhesion. No enlarged lymph nodes were found in the abdominal cavity.
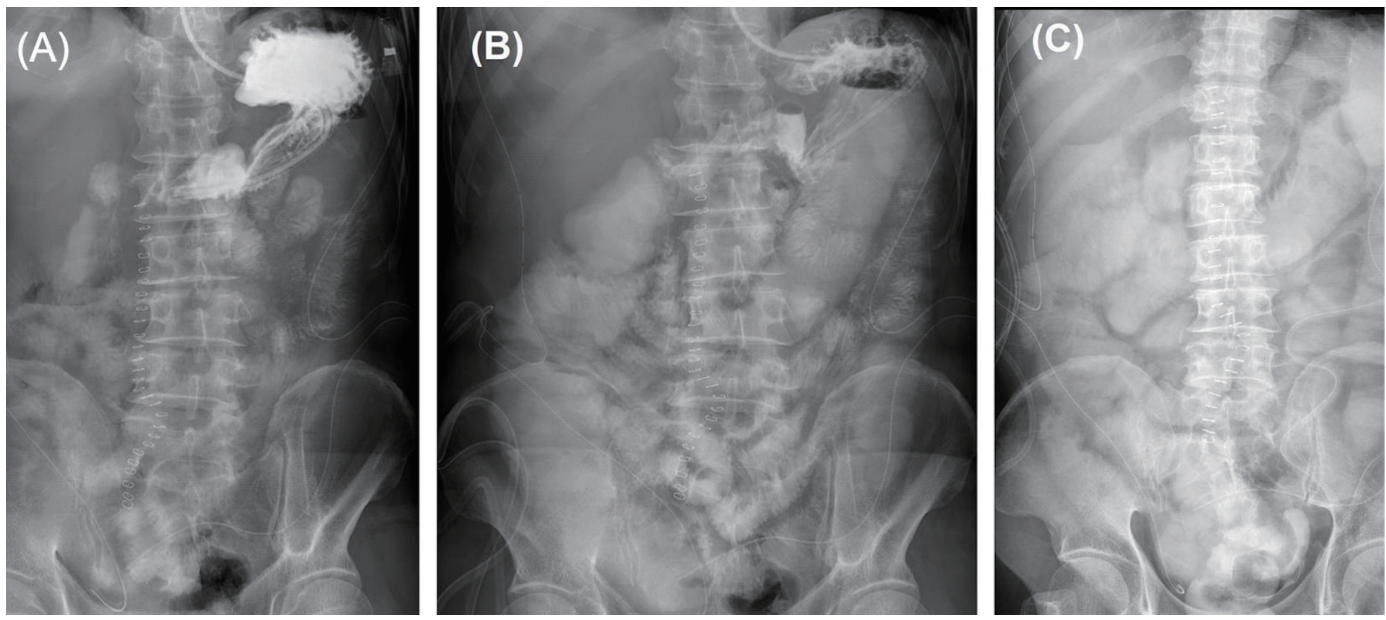

Fig. 3. Small bowel series: (A) after 2 hrs, (B) after 3 hrs, and (C) after 1 day. Successive inspections showed that majority of the contrast was retained in the intestine and some descended into the rectum. 


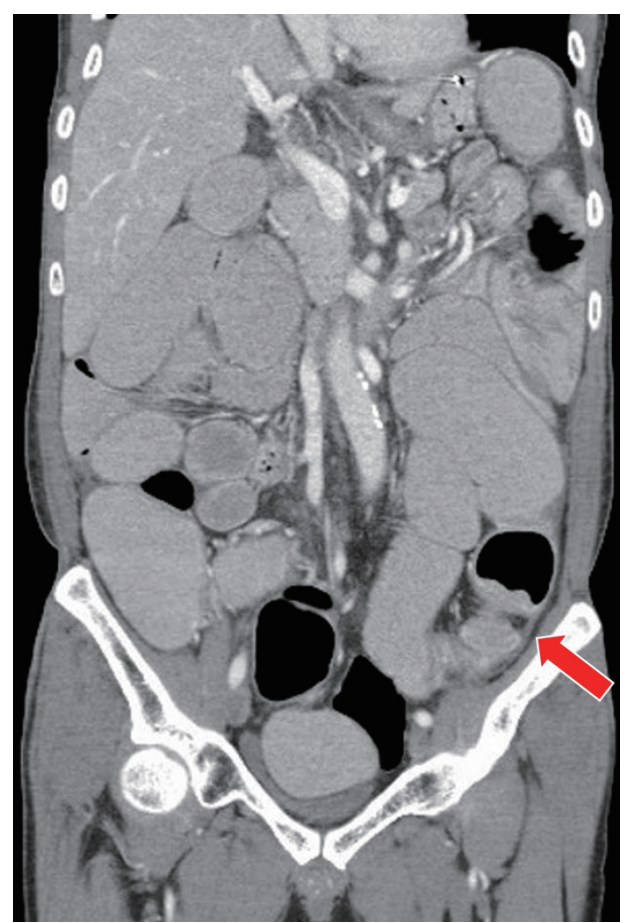

Fig. 4. Follow-up abdominal computed tomography. A masslike lesion with an apple core sign was seen at the descendingsigmoid colon junction site.

Two weeks after the operation, the patient's symptoms did not improve; hence, tests for intestinal obstruction were performed. It was confirmed by the small bowel series that part of the intestinal contents was passed (Fig. 3). The narrowing site identified in the previous ACT was seen as the apple core sign in this ACT (Fig. 4). In the sigmoidoscope, a cancer-like lesion obstructed the lumen entirely (Fig. 5); hence, loop transverse colostomy was performed. The patient's symptoms improved, and the biopsy result of the sigmoidoscope was an adenocarcinoma with moderate differentiation. The patient was managed with neoadjuvant chemotherapy and underwent left hemicolectomy five months later.

\section{Discussion}

Adhesion, increased hernia, and large bowel cancer are the main causes of total bowel obstructions $(3,5)$. Among them, adhesion is the most common factor and can interfere with the evaluation of other factors during surgery (14). About $60 \%$ of large bowel obstructions are cancers (4, 5). If there is a bowel obstruction, including the large bowel, in a patient without a previous abdominal operation

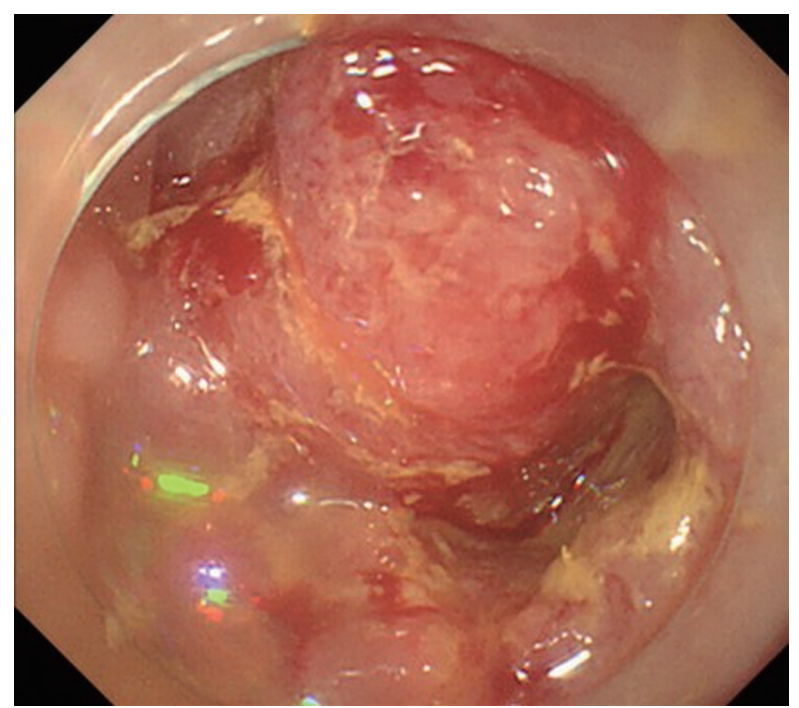

Fig. 5. Sigmoidoscopic image. A cancer-like lesion encircled the lumen.

history, the cause is likely to be a cancer. Whenever there is a large bowel obstruction, detailed history taking and evaluation to rule out cancer should be performed, even though the patient has experienced trauma-laparotomy.

\section{Conflict of interest}

No potential conflict of interest relevant to this article was reported.

\section{References}

1. Miller G, Boman J, Shrier I, Gordon PH. Natural history of patients with adhesive small bowel obstruction. Br J Surg. 2000;87(9):1240-7.

2. Wysocki A, Krzywoń J. Causes of intestinal obstruction. Przegl Lek. 2001;58(6):507-8.

3. Markogiannakis H, Messaris E, Dardamanis D, Pararas N, Tzertzemelis D, Giannopoulos P, et al. Acute mechanical bowel obstruction: clinical presentation, etiology, management, and outcome. World J Gastroentrerol. 2007;13(3):432-7.

4. Jaffe T, Thompson WM. Large-bowel obstruction in the adult: classic radiographic and CT findings, etiology, and mimics. Radiology. 2015;275(3):651-63.

5. Biondo S, Parés D, Frago R, Martí-Ragué J, Kreisler E, De Oca J, et al. Large bowel obstruction: predictive factors for postoperative mortality. Dis Colon Rectum. 2004;47(11):1889-97. 